\title{
Risk factors for the recurrence of an intracranial saccular aneurysm following endovascular treatment
}

\author{
De-Zhang Huang ${ }^{1}$, Bin Jiang ${ }^{1}$, Wei He ${ }^{1}$, Yi-Hua Wang ${ }^{1}$, Zhi-Gang Wang ${ }^{1}$ \\ ${ }^{1}$ Department of Neurosurgery, Qilu Hospital of Shandong University, Qingdao 266035, China \\ Correspondence to: Zhi-Gang Wang, email: wzg1110@126.com \\ Keywords: intracranial saccular aneurysm, tumor recurrence, retrospective study \\ Received: June 29, $2016 \quad$ Accepted: March 24, $2017 \quad$ Published: April 06, 2017 \\ Copyright: Huang et al. This is an open-access article distributed under the terms of the Creative Commons Attribution License (CC-BY), \\ which permits unrestricted use, distribution, and reproduction in any medium, provided the original author and source are credited.
}

\section{ABSTRACT}

Background: This study was aimed to determine risk factors for the recurrence of an intracranial saccular aneurysm (ISA) following endovascular treatment. The findings will help medical professionals to identify patients having a high risk of ISA recurrence and assist them in developing appropriate follow-up plans.

Results: During the follow-up period, $12.6 \%$ of the patients $(83 / 658)$ experienced recurrent ISAs. An analysis of related factors, including gender, age, hypertension, diabetes mellitus, smoking, tumor size, width of the aneurysm neck, the presence or absence of a rupture, the volume embolization ratio (VER), the application or nonapplication of a stent, and follow-up time, revealed that a tumor size $>10 \mathbf{~ m m}$ in diameter, wide-necked aneurysms, an anterior communicating or middle cerebral artery aneurysm, an aneurysm rupture, a VER $<20 \%$, the absence of stent assistance, and follow-up time were high-risk factors for the recurrence of ISAs.

Materials and Methods: We retrospectively reviewed the records of 658 patients who underwent endovascular treatment for ISAs from January 2010 through December 2014. Multivariable logistic regression was performed on the candidates' risk factors, which were identified via univariable screening analysis.

Conclusions: Smoking, a large tumor size, a wide-necked aneurysm, an anterior communicating or middle cerebral artery aneurysm, an aneurysm rupture, a VER $<20 \%$, and an absence of stent assistance are significant risk factors for the postoperative recurrence of an aneurysm. Strict follow-up plans should be created for ISA patients having these high-risk factors.

\section{INTRODUCTION}

An intracranial saccular aneurysm (ISA), which is characterized by high morbidity, mortality, and relapse, is the leading cause of spontaneous subarachnoid hemorrhage (SSAH). The overall morbidity and mortality rates associated with SSAH are $2 \times 10^{-5}-16 \times 10^{-5}$ and $8 \%-67 \%$ per year, respectively. With the development of imaging technology, the ISA detection rate has been gradually increasing. Microsurgery clipping and endovascular treatment have been the two main therapeutic treatments for ISA. With the rapid development of new materials and transcatheter arterial embolization, however, endovascular treatment has become the main treatment method for ISA [1]. Although endovascular treatment has lower fatality and disability rates compared with microsurgery clipping, its postoperative ISA recurrence rate is $15 \%-33 \%$, while that of microsurgery clipping is only $4 \%-14.7 \%$ [2-4]. Moreover, the high ISA recurrence rate associated with endovascular treatment has been its main challenge [1]. It is therefore important to identify the risk factors associated with aneurysm recurrence.

A number of studies have determined a variety of risk factors, including confounders (e.g., diabetes mellitus, hypertension, and smoking) and aneurysm-specific factors (e.g., tumor size, width of the aneurysm neck, and terminal or sidewall lesion) that affect the recurrence of a ruptured intracranial aneurysm. However, no systemic studies to date have been able to identify risk factors for aneurysm recurrence. A strict preoperative assessment should be performed, and a detailed treatment plan should be created, for patients with high risk factors in an effort to prevent aneurysm recurrence. Accordingly, the records of 658 patients who underwent endovascular treatment for ISA 
from January 2010 to December 2014 were retrospectively analyzed to determine the risk factors associated with ISA recurrence.

\section{RESULTS}

A total of 658 patients were included in this study (demographic characteristics are shown in Table 1). During the follow-up period, $12.6 \%$ of the patients (83/658) experienced ISA recurrence.

The univariate analyses of the potential predictors of the recurrence risk were performed. The predictors of recurrence risk identified by this method include gender, age, hypertension, diabetes mellitus, smoking, tumor size, width of the ISA neck, tumor site, presence or absence of rupture, the volume embolization ratio (VER), the application or nonapplication of a stent, and follow-up time (Table 1). The recurrence rate increased with cases in which the diameter of the original ISA was large. Pairwise comparisons showed that the $>10 \mathrm{~mm}$ group had a much higher recurrence rate than that of the $\leq 5-\mathrm{mm}$ group and the 5-10-mm group ( $p=0.0020, \chi^{2}=9.5551 ; p=8.1676$, and $\chi^{2}=0.0043$, respectively); however, there was no difference in recurrence between the $\leq 5-\mathrm{mm}$ group and the 5-10-mm group ( $p=0.6520, \chi^{2}=0.2034$; Table 1). The width of ISA necks and tumor sizes in different ISA locations were also compared. The results showed that the rate of wide-necked ISAs was significantly higher in anterior communicating artery ISAs and in middle cerebral artery ISAs (Table 2); meanwhile, the rate of tumors whose size was more than $10 \mathrm{~mm}$ in diameter was significantly higher in Middle cerebral artery ISAs (Table 3). The location of the ISA was also associated with the risk of recurrence. Pairwise comparisons demonstrated that the recurrence risk was similar among posterior circulation ISAs, posterior communicating ISAs, anterior cerebral artery ISAs, and other internal carotid artery ISAs $\left(p=0.4747, \chi^{2}=0.5109\right)$. The recurrence risk of anterior communicating artery ISAs and middle cerebral artery ISAs was different from that of the other groups $(p<0.05$; Table 1). Study findings also demonstrated that ISA rupture, VER $(<20 \%)$, and the absence of stent assistance were associated with recurrence (Table 1). The VER of the non-ruptured ISA was higher than ruptured ISAs ( $p=0.0027, \chi^{2}=8.9997$, Table 4$)$. More stents were applied in non-ruptured ISAs $\left(p=0.0018, \chi^{2}=9.7945\right.$, Table 5). It is interesting to note that follow-up time was found to be related to the postoperative recurrence of ISAs. The recurrence risk in six months was significantly different from other time periods $\left(p_{\leq 6 \text { vs } 6-12}=0.0025\right.$, $\left.p_{<6 \text { vs } 12-24}=0.0423, p_{<6 \text { vs }>24}=0.0048\right)$. Only $55.4 \%$ of recurrences occurred after six months, while $78.3 \%$ occurred after 12 months and $95.2 \%$ after 24 months (Table 1).

The multivariate logistic regression analysis (Table 6) revealed that the size of the tumor $(>10 \mathrm{~mm})$, wide-necked ISAs, anterior communicating or middle cerebral artery aneurysms, ISA rupture, VER $(<20 \%)$, and the absence of stent assistance are independently associated with ISA recurrence.

\section{DISCUSSION}

With the development of endovascular techniques in the treatment of both ruptured and nonruptured ISAs, it has become increasingly important to improve the obliteration efficacy. It was therefore essential to identify risk factors associated with the recurrence of ISAs and thereby enable medical professionals to formulate a more appropriate treatment plan. This analysis demonstrated that the tumor size of the ISA $(>10 \mathrm{~mm})$, wide-necked ISAs, anterior communicating or middle cerebral artery ISAs, ISA rupture, VER $(<20 \%)$, and the absence of stent assistance are risk factors for the recurrence of ISAs. These factors can be divided into three categories: ISA-specific factors, embolization-specific factors, and confounders, such as hypertension, smoking, and diabetes mellitus.

Various studies have demonstrated that tumor size is associated with the recurrence of ISAs. Raymond et al. found that ISA patients whose tumors have a diameter of more than $10 \mathrm{~mm}$ are more likely to develop the recurrence [5]. Furthermore, Campi et al. reported that the recurrence rate of ISAs with a tumor size larger than $10 \mathrm{~mm}$ was higher than that of ISAs with a tumor size less than $5 \mathrm{~mm}$ in diameter [6]. In this study, $25 \%$ of the examined ISA patients (16/63) with a tumor size larger than $10 \mathrm{~mm}$ in diameter experienced recurrence. Meanwhile, only $11.2 \%$ of the patients $(67 / 595)$ with a tumor size of less than 10 $\mathrm{mm}$ developed recurrence. These results suggest that ISAs are more likely to recur in cases where the tumor size is larger. The reason for this might be that a larger tumor size has a more irregular shape, which makes it difficult to perform dense filling. What's more, thrombosis often occurs in larger tumors, and this prevents the spring coil from occupying this area where the thrombus is. After the thrombosis is dissolved, the residual cavity will enlarge, consequently leading to recurrence.

According to previous reports, the neck width of the ISA is associated with its recurrence [7]. For example, Raymond et al. found that the recurrence rate of widenecked ISAs $(\geq 4 \mathrm{~mm})$ was as high as $52.3 \%$, while that of narrow-necked ISAs was only $23.7 \%$ during the follow-up period [5]. In the present study, 57/228 (25.0\%) patients with wide-necked ISAs experienced recurrences, while only $6.1 \%$ of the patients with narrow-necked ISAs developed recurrences $(p<0.001)$. This might be due to the difficulty in achieving dense filling in wide-necked ISAs. Wide-necked ISAs often have greater blood flow and complex stress gradients, which impact the filling effects of the spring coil [8]. Therefore, neck width was also found to be an independent risk factor for the recurrence of ISAs. 
Table 1: Univariate analysis of risk factors for ISA recurrence

\begin{tabular}{|c|c|c|c|c|c|}
\hline & \multirow[t]{2}{*}{ Risk Factors } & \multicolumn{2}{|c|}{ Recurrence } & \multirow[t]{2}{*}{$P$} & \multirow[t]{2}{*}{$\chi^{2}$} \\
\hline & & Yes & No & & \\
\hline \multicolumn{6}{|l|}{ Gender } \\
\hline & Male & 31 & 212 & 0.9325 & 0.0072 \\
\hline & Female & 52 & 363 & & \\
\hline \multicolumn{6}{|l|}{ Age } \\
\hline & $<60$ & 59 & 403 & 0.8527 & 0.0345 \\
\hline & $\geq 60$ & 24 & 172 & & \\
\hline \multicolumn{6}{|c|}{ Hypertension } \\
\hline & Yes & 77 & 495 & 0.0913 & 2.8521 \\
\hline & No & 6 & 80 & & \\
\hline \multicolumn{6}{|c|}{ Diabetes mellitus } \\
\hline & Yes & 26 & 180 & 0.9969 & 0.0000 \\
\hline & No & 57 & 395 & & \\
\hline \multicolumn{6}{|l|}{ Smoking } \\
\hline & Yes & 19 & 143 & 0.6958 & 0.1529 \\
\hline & No & 64 & 432 & & \\
\hline \multicolumn{6}{|l|}{ Rupture } \\
\hline & Yes & 77 & 479 & 0.0259 & 4.9625 \\
\hline & No & 6 & 96 & & \\
\hline \multicolumn{6}{|l|}{ Size } \\
\hline & $\leq 5 \mathrm{~mm}$ & 28 & 236 & 0.0052 & 10.5123 \\
\hline & $5-10 \mathrm{~mm}$ & 39 & 292 & & \\
\hline & $>10 \mathrm{~mm}$ & 16 & 47 & & \\
\hline \multicolumn{6}{|l|}{ Neck width } \\
\hline & $>4 \mathrm{~mm}$ & 57 & 171 & 0.0000 & 48.5581 \\
\hline & $\leq 4 \mathrm{~mm}$ & 26 & 404 & & \\
\hline & Other & 11 & 141 & & \\
\hline \multirow[t]{6}{*}{ Location } & & & & 0.0000 & 27.5219 \\
\hline & Posterior communicating ISA & 15 & 160 & & \\
\hline & Anterior communicating artery ISA & 35 & 122 & & \\
\hline & Anterior cerebral artery ISA & 2 & 41 & & \\
\hline & Middle cerebral artery ISA & 17 & 75 & & \\
\hline & Posterior circulation ISAs & 2 & 37 & & \\
\hline \multicolumn{6}{|l|}{ VER } \\
\hline & VER $\geq 20 \%$ & 32 & 325 & 0.0021 & 9.4344 \\
\hline & VER $<20 \%$ & 51 & 250 & & \\
\hline \multicolumn{6}{|l|}{ Stent } \\
\hline & Yes & 15 & 212 & 0.0008 & 11.3412 \\
\hline & No & 68 & 363 & & \\
\hline \multicolumn{6}{|c|}{ Follow-up time (Months) } \\
\hline & $\leq 6$ & 46 & 612 & 0.0013 & 15.6494 \\
\hline & $6-12$ & 19 & 575 & & \\
\hline & $12-24$ & 13 & 329 & & \\
\hline & $>24$ & 4 & 212 & & \\
\hline
\end{tabular}


Table 2: Proportion of wide-necked ISAs $(<4 \mathrm{~mm})$ at different locations of an intracranial artery

\begin{tabular}{lcccc}
\hline Location of ISAs & Wide neck & Narrow neck & Total & \% \\
\hline Anterior communicating artery & 62 & 95 & 157 & 39.5 \\
Middle cerebral artery & 48 & 44 & 92 & 52.2 \\
Other site & 118 & 291 & 409 & 28.9 \\
\hline
\end{tabular}

$\mathrm{p}_{\text {anterior vs middle }}=0.0517, \mathrm{p}_{\text {anterior vs other }}=0.0150, \mathrm{p}_{\text {middle vs other }}=0.0000$.

Table 3: The size of ISAs at different locations of an intracranial artery

\begin{tabular}{lcccc}
\hline Location & $\mathbf{1 0} \mathbf{~ m m}$ & $<\mathbf{1 0} \mathbf{~ m m}$ & Total & $\mathbf{\%}$ \\
\hline Anterior communicating artery & 16 & 141 & 157 & 10.2 \\
Middle cerebral artery & 21 & 71 & 92 & 22.8 \\
Other site & 26 & 383 & 409 & 6.4 \\
\hline
\end{tabular}

$\mathrm{p}_{\text {middle vs anterior }}=0.0068, \mathrm{p}_{\text {middle vs other }}=0.0000$.

The location of the ISA is another risk factor for recurrence. Posterior circulation ISAs are more likely to recur in patients receiving pure spring coil embolization: Ferns et al. reported that the recurrence rate in 862 patients with posterior circulation ISAs was $22.5 \%$ and that the recurrence rate in 1,901 patients with anterior circulation ISAs was $15.5 \%$ after endovascular treatment [9]. Teleb et al. came to a similar conclusion: The authors found that the recurrence rate in patients with posterior circulation ISAs was higher than that in patients with anterior circulation ISAs [10]. Because the case number was limited, we did not compare the recurrence rate between anterior circulation ISAs and posterior circulation ISAs in this study. However, we found that anterior communicating ISAs and middle cerebral artery ISAs are more likely to recur than other anterior circulation ISAs. This finding might be associated with hemodynamics, the parent vessel, and/or the structure of the branching vessels. First, the tumor direction of anterior communicating or middle cerebral artery ISAs mostly points to the blood flow of the parent artery. Even after endovascular treatment, the hemodynamics had not improved. Blood flow pressure and shear force are not altered persistently, thereby making it more easy to recur. Second, the branching vessels near the anterior communicating or middle cerebral artery are very important for ensuring vascular patency, which affects the surgeon's decision to embolize an ISA. Additionally, a larger tumor size in anterior communicating or middle cerebral artery ISAs was more often observed. In our study, $10.2 \%(16 / 157)$ of patients with anterior communicating ISAs had tumors more than $10 \mathrm{~mm}$ in diameter and $22.8 \%$ (21/92) of patients with middle cerebral ISAs had tumors more than $10 \mathrm{~mm}$ in diameter. However, only $6.4 \%$ of the patients had tumors more than $10 \mathrm{~mm}$ in diameter at other sites. The anterior communicating and middle cerebral artery ISAs also had a larger proportion of wide-necked ISAs (anterior communicating ISAs (39.5\%) versus middle cerebral ISAs $(52.2 \%)$ versus other ISAs $(28.9 \%)$ $(p<0.05)$.
Apart from ISA-specific factors, embolizationspecific factors, such as a dense degree of embolization, the application of stents, and the number of balloons and stents applied were also related to the recurrence of ISAs. A dense degree of embolization was measured by VER. In the present study, the VER $<20 \%$ group had a recurrence rate of $20.4 \%(51 / 250)$ compared with a $9.8 \%(32 / 325)$ recurrence rate in the VER $\geq 20 \%$ group. This finding was consistent with other similar studies $[6,11-13]$. Sluzewski et al. also found that no dense embolized patients developed recurrences, while $45.5 \%$ of the less dense embolized patients recurred after a six-month follow-up period [12]. Chalouhi et al.'s results showed that when the VER was below $22 \%$, the recurrence rate decreased with the increase of the embolization degree [13]. Campi et al. found that completely embolized ISAs required only $5.8 \%$ of retreatment but that incompletely or partially embolized ISAs had much higher recurrence rates (18.8\% and 20.6\%, respectively) [6]. This might be because the blood flow compresses the spring coil, and the thrombus is more easily dissolved when the VER is low, which enlarges the residual cavity. It was also reported that if there were remnants at the ISA neck, the remnants would be enlarged by the high shear stress and blood flow velocity [14]. Furthermore, the less dense embolization would lead to the formation of an unstable structure and displacements of the spring coil, both of which contribute to recurrence. Studies have demonstrated that the application of a stent could help to reduce recurrence rates [15]. In a study by Piotin et al., the application of a stent significantly reduced the recurrence rate (with stent, 14.9 versus without stent, $33.5 \% ; p<0.001$ ) [16], and Chalouhi et al. reached a similar conclusion [17]. The application of a stent can effectively improve the density of embolization and reconstruct the parental artery wall to enclose the ISA neck. Furthermore, a stent also improves hemodynamics and promotes intimal hyperplasia to cover the ISA neck $[15,17]$. These results showed that density of the initial 
Table 4: The VER of ruptured and non-ruptured ISAs

\begin{tabular}{lcccc}
\hline Rupture & $\mathbf{2 0 \%}$ & $<\mathbf{2 0 \%}$ & total & \% \\
\hline Yes & 281 & 275 & 556 & 50.5 \\
No & 68 & 34 & 102 & 66.7 \\
\hline
\end{tabular}

The VER of non-ruptured ISAs is higher than that of ruptured ISAs $\left(p=0.0027, \chi^{2}=8.9997\right)$.

Table 5: The application of a stent in ruptured and non-ruptured ISAs

\begin{tabular}{lcccc}
\hline Rupture & \multicolumn{2}{c}{ Stent } & total & \% \\
\cline { 2 - 4 } & Yes & No & & \\
\hline Yes & 178 & 378 & 556 & 32.0 \\
No & 49 & 53 & 102 & 48.0 \\
\hline
\end{tabular}

More stents were applied in non-ruptured ISAs $\left(p=0.0018, \chi^{2}=9.7945\right)$.

Table 6: Multivariate analysis of risk factors for ISA recurrence

\begin{tabular}{cccc}
\hline Risk factors & $\boldsymbol{P}$ & OR & $\mathbf{9 5 \%}$ C I \\
\hline size $>10 \mathrm{~mm}$ & 0.011 & 4.597 & $1.427-14.815$ \\
Wide-necked & 0.006 & 2.758 & $1.345-5.656$ \\
Anterior communicating artery & 0.001 & 1.195 & $1.072-1.331$ \\
Middle cerebral artery ISA & 0.004 & 1.099 & $1.030-1.173$ \\
Ruptured ISA & 0.006 & 4.038 & $1.481-11.010$ \\
VER $<20 \%$ & 0.019 & 4.284 & $1.276-14.382$ \\
Without stent & 0.001 & 1.205 & $1.076-1.349$ \\
\hline
\end{tabular}

embolization degree was one of the influential factors for the recurrence of the ISA embolization.

As the follow-up time is extended, the recurrence rate is also increased [6, 18]. Raymond held the opinion that a six-month follow-up is associated with only a $48 \%$ rate of recurrence [5]. In our study, a recurrence rate of only $55.4 \%$ occurred six months after endovascular treatment. Short-term angiographic follow-up could not fully determine the postoperative recurrence of ISAs. Because there are risks associated with performing cerebral angiography, it is necessary to prolong the followup time to at least 24-36 months.

The recurrence rate of ruptured ISAs was significantly higher than that of nonruptured ISAs [19]. In the present study, ruptured ISAs had a recurrence rate of $13.8 \%$, while the recurrence rate of nonruptured ISAs was only $5.9 \%(p<0.001)$; Raymond et al. obtained a similar finding in their study [5]. Moreover, ISA rupture plays a role in the promotion of blood clots. As time passes, the blood clotting is absorbed, leading to incomplete filling of the spring coil. In the present study, the VER of ruptured ISAs was lower than that of nonruptured ISAs. In addition, patients with ruptured ISAs cannot receive sufficient antiplatelet treatment, which limits stent assistance and further promotes recurrence [20].

\section{MATERIALS AND METHODS}

\section{Patient population}

We retrospectively reviewed the complete followup records of 658 patients who underwent endovascular treatment for ISA from January 2010 through December 2014. The demographic characteristics of the reviewed patients are summarized in Table 1.

\section{Criteria for inclusion}

- a diagnosis of ISA via digital subtraction angiography (DSA);

- the patient's first treatment consisted of an endovascular procedure, and the ISA was completely embolized, without residue;

- DSA angiographic follow-up records were complete (patients were followed up at least once and for more than six months postoperatively).

\section{Criteria for exclusion}

- the aneurysm was of a type other than ISA, such as a dissecting aneurysm or a fusiform aneurysm; 
- the patient's first treatment did not entail an endovascular procedure;

- the ISA was not completely embolized after the first endovascular treatment;

- patient records were incomplete, and the followup period was less than six months.

- patients with ISAs diagnosed at new-found sites.

\section{Definition of postoperative recurrence}

Angiographic results for re-examination demonstrated loosened or compressed spring coil, or contrast materials filling in the body or neck of the ISA, which was not observed on the immediate postoperative angiogram, when compared with the immediate postoperative angiographic results. The definition of recurrence was reviewed by three neuro-interventional experts.

\section{Surgical procedure}

Computed tomography angiography (CTA), magnetic resonance angiography (MRA), or DSA, was used to diagnose all the study patients with ISAs. First, a femoral artery puncture was performed using Seldinger's technique with the patient under general anesthesia, and a $6 \mathrm{~F}$ artery sheath was put into the artery. The ISA was then confirmed by DSA. Following the administration of systemic heparinization, the guiding catheter was guided to the petrous internal carotid artery or to the vertebral artery C2 segment using a 0.035 -inch guide wire. Three dimensional (3D) imaging was performed to further check the condition of the ISA. After choosing a working angle appropriate for obtaining 3D images, the microcatheter was inserted into the cavity of the ISA by micro-wire with the help of Road Map. If stent assistance was needed, the stent was sent to the distal side of the parent artery before the microcatheter was inserted into the ISA. Under the premise that the parent artery was unblocked, the ISA was embolized to the highest possible density. The embolization continued until angiography showed no contrast material in the tumor or neck. The patients were recommended to undergo DSA examinations 3 months, 9 months, 21 months, and 36 months after surgery.

\section{Data analysis}

Statistical analysis was performed using SPSS 13.0. Measurement data were expressed as mean \pm standard deviation (SD). Variables related to the recurrence of an ISA were analyzed by univariate analysis using Pearson's chi-squared test, the student's $t$-test, or the rank sum test. Multivariable logistic regression was performed on candidate predictor variables to identify independent risk factors for ISA recurrence. Statistical significance was defined as $p<0.05$.

\section{CONCLUSIONS}

In conclusion, in the present study, the overall postoperative recurrence rate of intracranial saccular aneurysms was $12.6 \%$. The following risk factors were found to be associated with the recurrence of ISAs: smoking, a large ISA tumor size, a wide-necked ISA, an anterior communicating or middle cerebral artery ISA, an ISA rupture, a VER $<20 \%$, and the absence of stent assistance were risk factors for the postoperative recurrence of ISA. Strict follow-up plans should be created for patients having high risk factors for recurrence. Because most recurrences occurred two years after surgery, the follow-up period should be extended to at least two years. Although the patients in the present study had relatively long-term follow-up compared with patients in other studies, it was a retrospective analysis with a limited case number of patients in only one medical center; further research is therefore necessary to address this limitation.

\section{CONFLICTS OF INTEREST}

The authors declare that they had no conflicts of interest.

\section{REFERENCES}

1. Molyneux A, Kerr R, Stratton I, Sandercock P, Clarke M, Shrimpton J, Holman R. International Subarachnoid Aneurysm Trial (ISAT) of neurosurgical clipping versus endovascular coiling in 2143 patients with ruptured intracranial aneurysms: a randomized trial. Journal of stroke and cerebrovascular diseases. 2002; 11:304-314.

2. Connolly ES Jr, Rabinstein AA, Carhuapoma JR, Derdeyn CP, Dion J, Higashida RT, Hoh BL, Kirkness CJ, Naidech AM, Ogilvy CS, Patel AB, Thompson BG, Vespa P, et al. Guidelines for the management of aneurysmal subarachnoid hemorrhage: a guideline for healthcare professionals from the American Heart Association/american Stroke Association. Stroke. 2012; 43:1711-1737.

3. Yoshimine T, Hashimoto N, Kawase T, Kirino T, Kobayashi S, Nagata I, Nukui H, Ogawa A, Ohmoto T, Saito I, Taneda M, Ueshima H, Yamada K, et al. Evidence-based guidelines for the management of aneurysmal subarachnoid hemorrhage. English Edition. Neurologia medico-chirurgica. 2012; $52: 355-429$.

4. Steiner T, Juvela S, Unterberg A, Jung C, Forsting M, Rinkel G. European Stroke Organization guidelines for the management of intracranial aneurysms and subarachnoid haemorrhage. Cerebrovascular diseases. 2013; 35:93-112.

5. Raymond J, Guilbert F, Weill A, Georganos SA, Juravsky L, Lambert A, Lamoureux J, Chagnon M, Roy D. Long-term angiographic recurrences after selective endovascular treatment of aneurysms with detachable coils. Stroke. 2003; 34:1398-1403. 
6. Campi A, Ramzi N, Molyneux AJ, Summers PE, Kerr RS, Sneade M, Yarnold JA, Rischmiller J, Byrne JV. Retreatment of ruptured cerebral aneurysms in patients randomized by coiling or clipping in the International Subarachnoid Aneurysm Trial (ISAT). Stroke. 2007; 38:1538-1544.

7. Dorfer C, Gruber A, Standhardt H, Bavinzski G, Knosp E. Management of residual and recurrent aneurysms after initial endovascular treatment. Neurosurgery. 2012; 70:537-553.

8. Luo B, Yang X, Wang S, Li H, Chen J, Yu H, Zhang Y, Zhang Y, Mu S, Liu Z, Ding G. High shear stress and flow velocity in partially occluded aneurysms prone to recanalization. Stroke. 2011; 42:745-753.

9. Ferns SP, Sprengers ME, van Rooij WJ, Rinkel GJ, van Rijn JC, Bipat S, Sluzewski M, Majoie CB. Coiling of intracranial aneurysms: a systematic review on initial occlusion and reopening and retreatment rates. Stroke. 2009; 40:523-529.

10. Teleb MS, Pandya DJ, Castonguay AC, Eckardt G, Sweis R, Lazzaro MA, Issa MA, Fitzsimmons BF, Lynch JR, Zaidat OO. Safety and predictors of aneurysm retreatment for remnant intracranial aneurysm after initial endovascular embolization. Journal of neurointerventional surgery. 2014; 6:490-494.

11. Leng B, Zheng Y, Ren J, Xu Q, Tian Y, Xu F. Endovascular treatment of intracranial aneurysms with detachable coils: correlation between aneurysm volume, packing, and angiographic recurrence. Journal of neurointerventional surgery. 2014; 6:595-599.

12. Sluzewski M, van Rooij WJ, Slob MJ, Bescos JO, Slump CH, Wijnalda D. Relation between aneurysm volume, packing, and compaction in 145 cerebral aneurysms treated with coils. Radiology. 2004; 231:653-658.

13. Chalouhi N, Dumont AS, Hasan D, Tjoumakaris S, Gonzalez LF, Starke RM, Dalyai R, El MS, Rosenwasser R, Jabbour P. Is Packing Density Important in Stent-Assisted Coiling? Neurosurgery. 2012; 71:386-387.
14. Meng H, Tutino VM, Xiang J, Siddiqui A. High WSS or low WSS? Complex interactions of hemodynamics with intracranial aneurysm initiation, growth, and rupture: toward a unifying hypothesis. American Journal of Neuroradiology. 2014; 35:1254-1262.

15. Hwang G, Park H, Bang JS, Jin SC, Kim BC, Oh CW, Kang HS, Han MH, Kwon OK. Comparison of 2-year angiographic outcomes of stent- and nonstent-assisted coil embolization in unruptured aneurysms with an unfavorable configuration for coiling. American Journal of Neuroradiology. 2011; 32:1707-1710.

16. Piotin M, Blanc R, Spelle L, Mounayer C, Piantino R, Schmidt PJ, Moret J. Stent-Assisted Coiling of Intracranial Aneurysms. Clinical and Angiographic Results in 216 Consecutive Aneurysms. 2010; 41:110-115.

17. Chalouhi N, Jabbour P, Singhal S, Drueding R, Starke RM, Dalyai RT, Tjoumakaris S, Gonzalez LF, Dumont AS, Rosenwasser R. Stent-assisted coiling of intracranial aneurysms: predictors of complications, recanalization, and outcome in 508 cases. Stroke. 2013; 44:1348-1353.

18. Lanzino G. The barrow ruptured aneurysm trial. Journal of neurosurgery. 2012; 116:133-134.

19. Raper DM, Allan R. International subarachnoid trial in the long run: critical evaluation of the long-term follow-up data from the ISAT trial of clipping vs coiling for ruptured intracranial aneurysms. Neurosurgery. 2010; 66:1166-1169.

20. Chalouhi N, Ali MS, Jabbour PM, Tjoumakaris SI, Gonzalez LF, Rosenwasser RH, Koch WJ, Dumont AS. Biology of intracranial aneurysms: role of inflammation. Journal of Cerebral Blood Flow \& Metabolism. 2012; 32:1659-1676. 